\title{
Carrier relaxation dynamics in InP quantum dots studied by artificial control of nonradiative losses
}

\author{
Ivan V. Ignatiev and Igor E. Kozin \\ Single Quantum Dot Project, ERATO, JST, 5-9-9 Tokodai, Tsukuba 300-2635, Japan \\ and Institute of Physics, St. Petersburg State University, St. Petersburg 198904, Russia \\ Selvakumar V. Nair, Hong-Wen Ren, and Shigeo Sugou \\ Single Quantum Dot Project, ERATO, JST, 5-9-9 Tokodai, Tsukuba 300-2635, Japan \\ Yasuaki Masumoto \\ Single Quantum Dot Project, ERATO, JST, 5-9-9 Tokodai, Tsukuba 300-2635, Japan \\ and Institute of Physics, University of Tsukuba, Tsukuba 305-8571, Japan
}

(Received 2 March 2000)

\begin{abstract}
A method to study carrier relaxation dynamics based on the artificial control of nonradiative losses by an external electric field is proposed. Clear evidence of phonon-assisted relaxation as the main relaxation mechanism of hot electron-hole pairs in InP self-assembled quantum dots is found by applying the proposed method. Efficient one-step relaxation processes with emission of acoustic and optical phonons are observed. These findings give important insights into the interaction of the electron-hole pairs in quantum dots with the phonon subsystem.
\end{abstract}

Quantum dots (QD's) are attractive for their atomiclike energy spectra that can be tailored by varying the size and shape of the QD. Because of the discrete energy spectrum, the carrier relaxation dynamics in QD's are significantly different from those in systems with continuous energy spectra. Although a thorough understanding of the carrier relaxation process is of prime importance, presently there are several seemingly contradictory reports on this topic and no clear description has emerged.

Generally two main mechanisms of relaxation are considered. The first one is the phonon assisted relaxation when a hot carrier relaxes to its ground state with emission of one or few phonons. However, theoretical analysis predicts that relaxation with longitudinal-optical (LO) phonon emission is possible only for a narrow energy window for the interlevel spacing and that the relaxation by emission of acoustic phonons, though possible for all energy level spacings, is very slow. ${ }^{1-3}$ Most experimental results contradict this prediction. The alternative mechanism is fast relaxation due to carrier-carrier collisions. ${ }^{4-6}$ This mechanism is assumed to be efficient when several carriers are present in the QD. If only one electron-hole pair is present in the QD, the final step of the relaxation should be again phonon-assisted relaxation of at least one of the carriers.

Conventional experimental techniques do not allow a direct proof of these mechanisms. Observation of resonances shifted from the excitation line by the LO phonon energy in the photoluminescence (PL) and photoluminescence excitation (PLE) spectra by several groups ${ }^{7-10}$ has been considered as experimental evidence of resonant LO phonon-assisted relaxation. However acoustic phonon resonances were not observed. On the other hand, kinetics measurement like timeresolved PL spectroscopy ${ }^{9,11}$ and differential transmission spectroscopy ${ }^{12}$ reveal fast carrier relaxation in few tens of picoseconds or less independent of the Stokes shift. This contradiction clearly shows that further experimental efforts are needed to clarify the relaxation mechanism.

In this paper we propose a powerful method to study the carrier relaxation in QD's exploiting the phenomenon of PL suppression when an external electric field is applied to a sample. ${ }^{13,14}$ We found that different resonances shifted from the excitation line by the LO or acoustic-phonon energy appear in the spectra of the partially suppressed PL. We explain this phenomenon as a result of the competition between nonradiative losses and intraband carrier relaxation processes. The rate of nonradiative losses may be controlled artificially by changing the applied bias. This opens up wide possibilities for the study of carrier relaxation dynamics. We checked the validity of the proposed method by independent measurements of the PL kinetics. By this method, we found direct evidence for efficient phonon-assisted carrier relaxation in self-assembled QD's. In particular, the relaxation is fairly efficient even with the emission of the high-energy acoustic phonons. This phenomenon offers challenges for theoretical analysis.

A heterostructure with one layer of InP self-assembled quantum dots embedded between $\mathrm{Ga}_{0.5} \operatorname{In}_{0.5} \mathrm{P}$ barrier layers was studied. The areal density of the QD's is about $10^{10} \mathrm{~cm}^{-2}$. Average base diameter is about $40 \mathrm{~nm}$ and the height is about $5 \mathrm{~nm}$. The sample was grown by gas source molecular-beam epitaxy on an $n^{+}$GaAs substrate. The total thickness of the nondoped epitaxial layer is $500 \mathrm{~nm}$. The sample was provided with a semitransparent gold Shottky contact (thickness $\approx 20 \mathrm{~nm}$ ) on the top surface and an Ohmic contact on the back surface.

We studied the last step of relaxation of carriers to their ground states because this step mostly determines the efficiency of the relaxation. For this purpose, the PL of the sample was excited selectively by a continuous wave (cw) Ti:sapphire laser within or slightly above the PL band of the 




FIG. 1. The PL (a) and PLE (b) spectra of InP QD's at the various applied bias indicated near each spectrum. The spectra are shifted and scaled for clarity. The spectral positions of the excitation (for PL) and of PL detection (for PLE) are shown by the vertical-dashed lines. Arrows indicate the spectral points LA and 2AC where the PL kinetics shown in Fig. 2(b) were measured.

QD's (hereafter referred to as quasiresonant excitation). The pump power density was typically less than $100 \mathrm{~W} / \mathrm{cm}^{2}$. A 1-m double monochromator and a photon counting system were used for the detection of the PL signal. We also measured the PL kinetics by using a mode-locked Ti:sapphire laser, a streak camera, and a $0.25-\mathrm{m}$ double subtractive dispersion monochromator. The pulse duration was $2 \mathrm{ps}$, and the pulse repetition rate was $82 \mathrm{MHz}$. To prevent the creation of more than one electron-hole pair in a QD, we used a fairly low pump power density of about $50 \mathrm{~W} / \mathrm{cm}^{2}$. The experiments were done at the sample temperature $2 \mathrm{~K}$.

The optical and transmission electron microscopy characterization of the sample studied is presented elsewhere. ${ }^{15}$ The energy spacing for the lowest electronic states is about 40 $\mathrm{meV}$ and that for holes is several $\mathrm{meV}$. Inhomogeneous broadening is about $50 \mathrm{meV}$, that is, larger than these values.

The PL bands of the QD's recorded under nonresonant (barrier band-to-band) and quasiresonant excitation have a smooth profile without any sharp features at zero bias. This behavior is typical for high-quality samples. When a negative bias is applied to the sample surface, the intensity of the PL decreases and distinct sharp resonances appear in the PL spectra [Fig. 1(a)]. Most prominent resonances are shifted from the excitation line approximately by the LO phonon energy of InP bulk crystal $[\hbar \omega=43.5 \mathrm{meV}$ (Ref. 16)]. PLE spectra reveal very similar behavior with prominent $1 \mathrm{LO}$ and 2LO resonances at negative bias as shown in Fig. 1(b).

The spectral positions of the LO resonances follow the excitation line. A plot of the integral intensity of the LO resonances versus their spectral positions reflects the PL profile of the QD's in the absence of bias. The LO resonances become hardly seen with increasing of the sample temperature above $60 \mathrm{~K}$. All experimental data strongly suggest that the observed resonances are caused by fast relaxation of hot carriers with emission of LO phonons rather than by phonon sidebands of the resonance PL or by resonant Raman scattering.



FIG. 2. (a) The PL kinetics at the LO resonance under various applied bias indicated near each curve. (b) Initial part of PL kinetics at zero bias at spectral points corresponding to one (LA) and two (2AC) acoustic phonon and LO phonon (LO) assisted relaxation marked in Fig. 1(a). The laser pulse (dashed curve) with full width at half maximum of 6 ps indicates the time resolution of the setup.

A similar phenomenon is observed also in the PL spectra of $\mathrm{In}_{0.35} \mathrm{Ga}_{0.65}$ As quantum dots with PL maximum at 1200 $\mathrm{meV} .{ }^{17} \mathrm{~A}$ few strong phonon resonances with Stokes shifts of $19 \mathrm{meV}, 29.5 \mathrm{meV}$, and $35.5 \mathrm{meV}$ appear at $U_{\text {bias }}<-2$ $\mathrm{V}$. These can be assigned to the carrier relaxation with emission of longitudinal acoustic (LA) phonons and InAs-like and GaAs-like LO phonons, respectively.

Our explanation for the observed phenomenon may be summarized as follows. The applied electric field activates a process of nonradiative losses of the QD excitation. The most likely cause of these losses in the structure with InP QD's is the tunneling of the hole from the QD into the barrier layer because of the small depth of the potential well for holes in such heterostructures. ${ }^{18}$ The details of this process will be discussed elsewhere. ${ }^{19}$ The tunneling from the excited state competes with relaxation to the ground state. This leads to selective PL suppression because of different relaxation rates with emission of $\mathrm{LO}$ and acoustic phonons.

The LO phonon assisted relaxation is possible in those QD's where the interlevel energy spacing $\Delta E$ coincides with the LO phonon energy $E_{L O}$. Selective laser excitation creates carriers in a subset of QD's. However, the shape as well as size distribution of the QD's gives rise to inhomogeneous broadening of the interlevel spacing. So the requirement $\Delta E=E_{L O}$ can be always fulfilled for some subset of QD's independently of the photon energy of excitation. ${ }^{7-10}$

To verify the assumptions implicit in the above description, we performed PL kinetics measurements. Kinetics for selected spectral points and their dependence on the applied bias for the LO resonance are shown in Fig. 2. A clear short- 


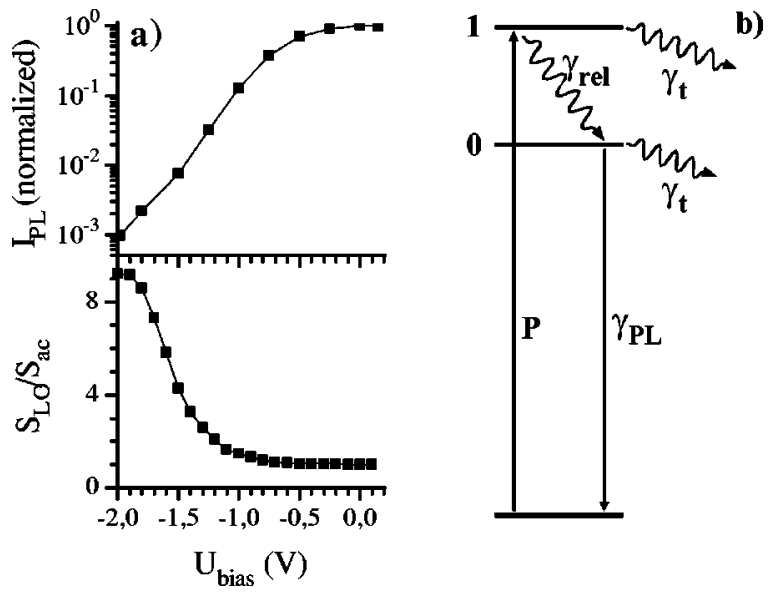

FIG. 3. (a) The integral intensity of the PL spectra (top curve) and the ratio $S_{L O} / S_{a c}$ (bottom curve) versus $U_{\text {bias }} . S_{L O}$ and $S_{a c}$ are the averaged intensities of $\mathrm{LO}$ resonance and $2 \mathrm{AC}$ spectral point marked in Fig. 1(a). The ratio is normalized to unity at zero bias. The solid lines are guides to the eye. (b) A schematic illustration of the three level model used for the analysis of the bias dependence of the PL.

ening of the decay time with the increase of negative bias is observed. So the nonradiative process forms some kind of time (or optical) gate for the PL. Initial (rise) part of the kinetics provides information about the relaxation rates. As seen from Fig. 2(b), it is very fast for the LO resonance and limited by our time resolution of about 6 ps. In the spectral region formed by the acoustic-phonon assisted relaxation, the rise time is about $50 \mathrm{ps}$, which is much longer than that at the $\mathrm{LO}$ resonance. ${ }^{20}$

Based on the above description, we can now understand the behavior of the time integrated spectra measured in the presence of the electric field. In Fig. 3(a) we plotted the bias dependence of the integral PL intensity and also the ratio of the intensities of the LO resonance and 2AC spectral point as a measure of the contrast of the features. A simple three level model, shown schematically in Fig. 3(b), can be used to analyze the one-step relaxation with emission of a single phonon. In this case, only the initial state " 1 " of the carriers created by selective excitation and the final radiative state " 0 " are involved in the process.

The dynamics of the populations $n_{1}$ and $n_{0}$ of the states " 1 " and " 0, ," respectively, is described by

$$
\begin{gathered}
\frac{d n_{1}}{d t}=P-\left(\gamma_{r e l}+\gamma_{t}\right) n_{1}, \\
\frac{d n_{0}}{d t}=\gamma_{r e l} n_{1}-\left(\gamma_{P L}+\gamma_{t}\right) n_{0} .
\end{gathered}
$$

Here $P$ is the rate of optical pumping, $\gamma_{r e l}$ is the relaxation rate, and $\gamma_{P L}$ is the radiative recombination rate. We model the nonradiative losses (e.g., hole tunneling) from the states " 1 " and " 0 " by a single rate $\gamma_{t}$ for simplicity.

For a cw excitation ( $P=$ const.), the solution of Eq. (1) yields the PL intensity $I_{P L}$ as

$$
I_{P L}=\gamma_{P L} n_{0}=P \frac{1}{1+\left(\gamma_{t} / \gamma_{P L}\right)} \frac{1}{1+\left(\gamma_{t} / \gamma_{r e l}\right)} .
$$



FIG. 4. (a) The PL spectrum at $U_{\text {bias }}=-1.5 \mathrm{~V}$ and cw excitation (solid line) and the time resolved PL spectrum reconstructed from kinetics data (circles) as described in the text. Solid line through the circles is a guide to the eye. "TA," "LA," "LA'," and 'LO' mark the phonon resonances. (b) Phonon density-ofstates of the InP crystal (Ref. 21).

A few cases can be considered depending on the ratio of the various relaxation rates.

Case 1. When the tunneling is slow, that is $\gamma_{t} \ll \gamma_{\text {rel }}$, Eq. (2) is reduced to $I_{P L}=P /\left[1+\left(\gamma_{t} / \gamma_{P L}\right)\right]$. It is seen that the PL intensity does not depend on the relaxation rate $\gamma_{\text {rel }}$. The PL band has a smooth shape without any phonon resonances. This case corresponds to the curves in Fig. 3(a) for small bias.

Case 2. When the tunneling is fast enough so that $\gamma_{L O}$ $>\gamma_{t}>\gamma_{a c}$, the PL suppression is stronger in the case of the slow acoustic-phonon assisted relaxation $\gamma_{r e l}=\gamma_{a c}$, than in the case of the fast LO phonon assisted relaxation $\gamma_{\text {rel }}$ $=\gamma_{L O}$. The ratio of the PL intensities $I_{L O}$ and $I_{a c}$ in the spectral regions formed by the LO phonon and acoustic phonon-assisted relaxation, respectively, is given by $I_{L O} / I_{a c}=\left[1+\left(\gamma_{t} / \gamma_{a c}\right)\right] /\left[1+\left(\gamma_{t} / \gamma_{L O}\right)\right]$. This ratio can be much more than unity because $\gamma_{L O} \gg \gamma_{a c}$. This case corresponds to the curves in Fig. 3(a) for bias in the range $-1.8 \mathrm{~V}<U_{\text {bias }}<-1 \mathrm{~V}$.

Case 3. In the case of extremely fast tunneling $\gamma_{t}$ $>\gamma_{L O}$, the ratio of the intensities $I_{L O} / I_{a c}=\gamma_{L O} / \gamma_{a c}$, that is the ratio depends only on the phonon-assisted relaxation rates. This case corresponds to the curves in Fig. 3(a) for bias $U_{\text {bias }}<-1.8 \mathrm{~V}$.

It is clear from the above analysis that the manipulation of the nonradiative losses allows us to determine spectral dependence of the relaxation rate. To demonstrate it, we measured the PL kinetics at many spectral points at zero bias, then multiplied them by $e^{-\gamma_{t} t}$ to imitate the fast nonradiative losses and integrated. The time resolved PL spectrum reconstructed this way is shown in Fig. 4(a) together with the PL spectrum measured at $U_{\text {bias }}=-1.5 \mathrm{~V}$. As the estimated tunneling time at $-1.5 \mathrm{~V}$ is smaller than the time resolution of our setup, $\tau_{s}=6 \mathrm{ps}$, we have used $\gamma_{t}=1 / \tau_{s}$. The excellent agreement between the two spectra clearly demonstrates the consistency of our description of the PL process. At the same 
time, the spectral and effective time resolutions in the PL spectrum of the biased sample is higher than that achievable in kinetics experiments.

A few important statements about relaxation mechanisms and relaxation rates can be derived from the experimental data and the analysis presented above.

The main relaxation mechanism of hot carriers in InP QD's, when only one electron-hole pair is created in the QD, is the phonon assisted relaxation. Different relaxation channels are possible. Electron and hole may relax separately or together, mediated by the Coulomb interaction, by the emission of phonons. The PL kinetics for the LO resonance shows mainly a fast rise component. From this kinetics it follows that single LO phonon emission contributes more than $70 \%$ of the PL at the LO resonance energy.

The relaxation time with emission of $\mathrm{LO}$ phonons lies in the range $0.6 \mathrm{ps}<\tau_{L O}<6 \mathrm{ps}$. The lower limit is derived from fitting the peaks in the LO resonance by Lorenzians. The upper limit is decided by the time resolution of the direct kinetics measurements.

Acoustic phonon resonances are observed in the spectra of the partially suppressed PL. A comparison of the PL spectrum with the phonon density-of-states (DOS) of InP crystal (see Fig. 4) allows us to attribute the resonances to the transverse acoustic (TA) and longitudinal acoustic (LA) peaks in the phonon DOS of InP (Ref. 21) and $\mathrm{Ga}_{0.5} \mathrm{In}_{0.5} \mathrm{P} .{ }^{22}$ The rising kinetics of the PL in these peaks are faster than in the rest of the acoustic part of the spectrum. This observation is evidence of efficient relaxation of electron-hole pairs with emission of high-frequency acoustic phonons, in stark contrast with theoretical predictions. ${ }^{1-3}$

In conclusion, we developed a method for the study of carrier relaxation in QD's based on the artificial control of the nonradiative losses by an external electric field. The nonradiative process with a controllable rate gives rise to an effective optical gate with variable duration for the PL of the QD's. This method allows one to study the spectral dependence of the carrier relaxation rates with high spectral and time resolution at a low-power density of optical excitation. Our results clearly demonstrate that the main relaxation mechanism in InP self-assembled QD's is the phononassisted relaxation. Clear evidence of efficient one-step relaxation with emission of high-frequency acoustic phonons is found. This observation poses a principal problem for theoretical analysis.

We thank Professor D. N. Talwar for kindly providing us the unpublished data on the phonon density-of-states of InP. We also thank V. Davydov for technical assistance in the experiments.
${ }^{1}$ U. Bockelmann and G. Bastard, Phys. Rev. B 42, 8947 (1990).

${ }^{2}$ H. Benisty, C. M. Sotomayor-Torrés, and C. Weisbuch, Phys. Rev. B 44, 10945 (1991).

${ }^{3}$ T. Inoshita and H. Sakaki, Phys. Rev. B 46, 7260 (1992).

${ }^{4}$ U. Bockelmann and T. Egeler, Phys. Rev. B 46, 15574 (1992).

${ }^{5}$ A. L. Efros, V. A. Kharchenko, and M. Rosen, Solid State Commun. 93, 281 (1995).

${ }^{6}$ S. Nair and Y. Masumoto, J. Lumin. (to be published); Phys. Status Solidi B (to be published).

${ }^{7}$ S. Fafard et al., Phys. Rev. B 52, 5752 (1995).

${ }^{8}$ R. Heitz et al., Appl. Phys. Lett. 68, 361 (1996).

${ }^{9}$ M. Vollmer et al., Phys. Rev. B 54, 17292 (1996).

${ }^{10}$ M. J. Steer et al., Phys. Rev. B 54, 17738 (1996).

${ }^{11}$ B. Ohnesorge et al., Phys. Rev. B 54, 11532 (1996).

${ }^{12}$ T. S. Sosnowski et al., Phys. Rev. B 57, R9423 (1998).

${ }^{13}$ K. H. Schmidt, G. Medeiros-Ribeiro, and P. M. Petroff, Phys. Rev. B 58, 3597 (1998).
${ }^{14}$ I. E. Kozin et al., J. Lumin. 87-89, 441 (2000).

${ }^{15}$ Y. Masumoto et al., Jpn. J. Appl. Phys., Part 1 38, 563 (1999).

${ }^{16}$ E. Bedel et al., J. Phys. C 19, 1471 (1986).

${ }^{17}$ V. G. Davydov, I. V. Ignatiev, J. S. Lee, S. V. Nair, K. Nishi, and Y. Masumoto (unpublished).

${ }^{18}$ C. Pryor, M.-E. Pistol, and L. Samuelson, Phys. Rev. B 56, 10 404 (1996).

${ }^{19}$ I. Kozin, I. Ignatiev, H.-W. Ren, S. Sugou, and Y. Masumoto (unpublished).

${ }^{20}$ The rise part of PL kinetics at acoustic resonances contains a small amount of fast component ( $\tau<6 \mathrm{ps}$ ). This component decreases with increasing negative bias and is hardly seen at $U_{\text {bias }}<-0.5 \mathrm{~V}$.

${ }^{21}$ D. N. Talwar and M. Vandevyver, Phys. Rev. B 41, 12129 (1990); (private communication).

${ }^{22}$ B. Ulrici and E. Jahne, Phys. Status Solidi B 86, 517 (1978). 